\title{
Chronic dietary supplementation of proanthocyanidins corrects the mitochondrial dysfunction of brown adipose tissue caused by diet-induced obesity in Wistar rats
}

\author{
David Pajuelo, Helena Quesada, Sabina Díaz, Anabel Fernández-Iglesias, Anna Arola-Arnal, \\ Cinta Bladé, Josepa Salvadó and Lluís Arola* \\ Nutrigenomics Group, Department of Biochemistry and Biotechnology, Universitat Rovira $i$ Virgili, Marcel li Domingo, \\ s/n. Campus Sescelades, 43007 Tarragona, Spain \\ (Received 16 February 2011 - Revised 12 April 2011 - Accepted 14 April 2011 - First published online 28 June 2011)
}

\begin{abstract}
The present study aims to determine the effects of grape seed proanthocyanidin extract (GSPE) on brown adipose tissue (BAT) mitochondrial function in a state of obesity induced by diet. Wistar male rats were fed with a cafeteria diet (Cd) for 4 months; during the last $21 \mathrm{~d}$, two groups were treated with doses of 25 and $50 \mathrm{mg}$ GSPE/ kg body weight. In the BAT, enzymatic activities of citrate synthase, cytochrome $c$ oxidase (COX) and ATPase were determined and gene expression was analysed by real-time PCR. The mitochondrial function of BAT was determined in fresh mitochondria by high-resolution respirometry using both pyruvate and carnitine-palmitoyl-CoA as substrates. The results show that the Cd causes an important decrease in the gene expression of sirtuin 1 , nuclear respiratory factor 1 , isocitrate dehydrogenase $3 \gamma$ and $\mathrm{COX} 5 \alpha$ and, what is more telling, decreases the levels of mitochondrial respiration both with pyruvate and canitine-palmitoyl-CoA. Most of these parameters, which are indicative of mitochondrial dysfunction due to diet-induced obesity, are improved by chronic supplementation of GSPE. The beneficial effects caused by the administration of GSPE are exhibited as a protection against weight gain, in spite of the Cd the rats were fed. These data indicate that chronic consumption of a moderate dose of GSPE can correct an energy imbalance in a situation of diet-induced obesity, thereby improving the mitochondrial function and thermogenic capacity of the BAT.
\end{abstract}

Key words: Mitochondria: Polyphenols: Proanthocyanidins: Obesity: Brown adipose tissue: Oxidative phosphorylation

Obesity is the result of a prolonged positive energy balance, which is produced by a greater amount of energy intake than expenditure ${ }^{(1)}$, and is a product of complicated interactions between an individual's genetic background and the immediate environment ${ }^{(2)}$. Obesity is widely recognised as one of the biggest global problems, not only to overall health, but also at an economic and social level. It is even considered an epidemic in some areas, especially developed countries, which suggests that our lifestyle plays an important role in obesity ${ }^{(2)}$. In this sense, it is important to consider strategies to reduce obesity.

Several studies performed in rodents have shown the antiobesity function of brown adipose tissue (BAT). It has been reported that surgical denervation or excision of BAT was accompanied by an abnormal increase in white adipose tissue deposits in those animals ${ }^{(3)}$. Indeed, in most rodent models, proneness to obesity reportedly correlates with decreased BAT activity, whereas resistance to obesity correlates with increased BAT function or the induction of brown adipocyte-like gene expression in white adipose tissue $e^{(3-5)}$. Recent findings ${ }^{(6-8)}$ provide evidence for the existence of metabolically active BAT depots in adult human subjects. Also, the activity of BAT has been published to be lower in overweight and obese individuals than in lean subjects ${ }^{(6)}$, making BAT a potentially interesting target for the treatment for obesity through the implementation of strategies that succeed in increasing the activity of BAT mitochondria. Strategies to increase the number and function of mitochondria involving nutrition and drugs have lately received increasing attention. Among the most effective are energy restriction, consumption of natural polyphenols, such as resveratrol, and drugs that have been shown to increase mitochondrial biogenesis via an increase in the transcriptional co-activator sirtuin 1 (Sirt1) and PPAR- $\gamma$ co-activator $(\mathrm{PGC} 1 \alpha)^{(9-11)}$.

Proanthocyanidins, a family of natural polyphenolic flavonoids, are present in a wide variety of plant foods, making them an important part of the human diet; they are found especially in fruits, berries, beans, nuts, cocoa and wine ${ }^{(12)}$.

Abbreviations: BAT, brown adipose tissue; Cd, cafeteria diet; COX, cytochrome $c$ oxidase; GSPE, grape seed proanthocyanidin extract; IDH3 $\gamma$, isocitrate dehydrogenase-3 $\gamma$; Nrf 1, nuclear respiratory factor 1; PGC1 $\alpha$, PPAR- $\gamma$ co-activator; Sirt1, sirtuin 1; UCP1, uncoupling protein 1. 
Proanthocyanidins have been shown to have beneficial effects on health; in fact, epidemiological studies have strongly suggested that regular consumption of proanthocyanidins may prevent the risk of $\mathrm{CVD}^{(13)}$. More specifically, proanthocyanidins have been described as antioxidants ${ }^{(14)}$, anti-inflammatory agents ${ }^{(15)}$ and they may even have hypolipidaemic effects ${ }^{(16)}$. Given the structural similarity of proanthocyanidins to resveratrol and other flavonoid derivatives that have been shown to increase mitochondrial biogenesis ${ }^{(9,11,17)}$, we hypothesised that grape seed proanthocyanidin extract (GSPE) would increase mitochondrial biogenesis in BAT. This increase in biogenesis would increase mitochondrial function and, consequently, increase energy expenditure, resulting in a possible method of fighting obesity through administration of GSPE. The purpose of the present study was to evaluate the role of long-term supplementation of GSPE on mitochondrial function and the thermogenesis capacity of BAT at a dose that is safe and practical for use as a dietary supplement in cafeteria-diet-induced obese rats.

\section{Materials and methods}

\section{Proanthocyanidin extract}

GSPE was kindly provided by Les Dérives Résiniques et Terpéniques (Dax, France). This proanthocyanidin extract contained monomeric (21.3\%), dimeric (17.4\%), trimeric $(16 \cdot 3 \%)$, tetrameric $(13 \cdot 3 \%)$ and oligomeric (5-13 units, $31 \cdot 7 \%)$ proanthocyanidins.

\section{Animals and experimental design}

A total of twenty-four, 6-week-old, male Wistar rats (Crl:WI (Han)) were purchased from Charles River (Barcelona, Spain). The rats were singly housed in cages in animal quarters at $22^{\circ} \mathrm{C}$ with a $12 \mathrm{~h}$ light $-12 \mathrm{~h}$ dark cycle (light from 08.00 to 20.00 hours) and were fed ad libitum with a standard chow diet (Panlab 04, Barcelona, Spain) and tap water. After 1 week of adaptation, the animals were randomly divided into four groups $(n$ 6). Of these, one group was considered the control group, and it was fed ad libitum with a standard chow diet; the other three groups were composed of a control cafeteria diet (Cd), Cd plus $25 \mathrm{mg}$ dose of GSPE/ $\mathrm{kg}$ and Cd plus $50 \mathrm{mg}$ dose of GSPE/ $\mathrm{kg}$, which were fed ad libitum with a standard chow diet plus a $\mathrm{Cd}$ as a model of a high-fat diet. The gross composition of the diets was as follows: for the standard chow diet, $60.5 \%$ carbohydrate, $2.9 \%$ lipid, $15.4 \%$ protein, $12 \%$ water, $5.3 \%$ minerals and $3.9 \%$ fibre, and for the Cd, $35.2 \%$ carbohydrate, $23.4 \%$ lipid, $11.7 \%$ protein, $28.4 \%$ water and $1.31 \%$ fibre $^{(18)}$. After 15 weeks, and while still maintaining the $\mathrm{Cd}$, the rats were trained to lick condensed milk voluntarily (composition per $100 \mathrm{~g}: 8.9 \mathrm{~g}$ protein, $0.4 \mathrm{~g}$ fat, $60.5 \mathrm{~g}$ carbohydrates, $1175 \mathrm{~kJ}$ (281 kcal)), which was used as a vehicle for administering GSPE. Both the control group and the control Cd group were given only condensed milk $(750 \mu \mathrm{l})$, and the other two groups were given the corresponding dose of GSPE per body weight mixed with the same volume of condensed milk. Treatment was administered every day at
09.00 hours for $21 \mathrm{~d}$. The last day of treatment, the rats were not fed to allow $12 \mathrm{~h}$ of fasting before killing the next day at 08.00 hours. The animals were killed after $12 \mathrm{~h}$ of fasting. Body weight was monitored weekly throughout the experiment. All experimental procedures were performed according to the national and institutional guidelines for animal care and use that are in place at our university. The Animal Ethics Committee of our university approved all procedures.

\section{Tissue collection}

The rats were anaesthetised with ketamine/xylazine and killed by exsanguination from the abdominal aorta using syringes, with heparin as the anticoagulant. Plasma was obtained by centrifugation and stored at $-80^{\circ} \mathrm{C}$ until analysis. Interscapular BAT was rapidly excised. Then, one sample of the BAT was frozen immediately in liquid $\mathrm{N}_{2}$ and stored at $-80^{\circ} \mathrm{C}$ until RNA extraction. Another part of the BAT was placed on ice and processed for mitochondrial isolation, as described by Fuster et $a l .{ }^{(19)}$, for use in enzymatic assays. The remaining BAT was rapidly processed using the mitochondrial isolation procedure according to Cannon \& Nedergaard ${ }^{(20)}$ for use in mitochondrial respirometry. Protein concentration in the mitochondrial pellet was measured using the fluorescamine (Fluram ${ }^{\circledR}$; Sigma, St Louis, MO, USA) method with bovine serum albumin as a standard ${ }^{(21)}$. Freshly isolated mitochondria were used immediately for mitochondrial respirometry. Protease inhibitor cocktail (P8340; Sigma) was added to the remaining mitochondrial samples, and they were stored at $-80^{\circ} \mathrm{C}$ for further analysis.

\section{Blood plasma assay}

Plasma glucose and TAG were measured with enzymatic colorimetric kits (QCA, Barcelona, Spain). Lactate concentration was determined with an enzymatic colorimetric kit (R-Biopharm AG, Roche, Germany). The levels of 3-hydroxybutyric acid were measured with an enzymatic colorimetric kit (IVD; BEN Srl, Italy). The manufacturer's protocol was followed in all cases.

\section{Oxidative phosphorylation in isolated mitochondria}

Mitochondrial isolation was carried out from the BAT according to Cannon \& Nedergaard ${ }^{(20)}$. To determine the functionality of oxidative phosphorylation in ex vivo mitochondria, we measured $\mathrm{O}_{2}$ consumption in different mitochondrial states polarographically, as described previously ${ }^{(20,22)}$, at $37^{\circ} \mathrm{C}$ using a two-chamber Oxygraph (Oroboros ${ }^{\circledR}$ Instruments; Innsbruck, Austria). Results are expressed as nmol $\mathrm{O}_{2} / \mathrm{mg}$ mitochondrial protein per min. The following two protocols were performed: (1) pyruvate as a substrate according to Cannon \& Nedergaard $^{(20)}$ and (2) carnitine-palmitoyl-CoA as a substrate according to Cannon ${ }^{(22)}$. All substrates used in these analyses were dissolved in Milli-Q water except oligomycin and carbonyl cyanide $P$-trifluoromethoxyphenylhydrazone, which were dissolved in $96 \%$ ethanol. 


\section{Enzymatic assays}

Mitochondrial extracts were isolated according to Fuster et al. ${ }^{(19)}$. Tissues were homogenised at $4^{\circ} \mathrm{C}$ in $1: 3(\mathrm{w} / \mathrm{v})$ of a buffer containing $250 \mathrm{~mm}$-sucrose, $1 \mathrm{~mm}$-EDTA and $10 \mathrm{~mm}$ Tris $-\mathrm{HCl}, \mathrm{pH} 7 \cdot 4$, using a Teflon/glass homogeniser, and then centrifuged at $700 \mathrm{~g}$ for $10 \mathrm{~min}$ at $4^{\circ} \mathrm{C}$. Then, the supernatant was centrifuged again at $12000 \mathrm{~g}$ for $10 \mathrm{~min}$ at $4^{\circ} \mathrm{C}$. The resulting pellet containing the purified mitochondria was resuspended in $100 \mu \mathrm{l}$ of a buffer with $70 \mathrm{~mm}$-sucrose, 220 mm-mannitol, 2 mm-HEPES and 1 mm-EDTA, pH 7·4.

Citrate synthase activity measurements were carried out as described by Srere ${ }^{(23)}$. The method uses a spectrophotometer to monitor changes in absorbance at $412 \mathrm{~nm}$ in the presence of 5,5'-dithiobis-(2-nitrobenzoate).

Cytochrome $c$ oxidase (COX) activity measurement was performed as described by Fuster et al ${ }^{(19)}$ in mitochondrial fractions (obtained as described previously) through a spectrophotometric method that monitors changes in absorbance at $550 \mathrm{~nm}$ in the presence of reduced substrates of cytochrome $c$ from ferrocytochrome $c$ (oxidised state; Sigma).

ATPase activity measurement was performed according to Penefsky \& Bruist $^{(24)}$ using a spectrophotometric method that monitors changes in absorbance at $339 \mathrm{~nm}$ in the presence of phosphoenolpyruvate, lactate dehydrogenase and pyruvate kinase, and other reagents. The reaction is linear and was monitored for at least $3 \mathrm{~min}$.

\section{Gene expression}

Total RNA from BAT was obtained using an RNeasy Lipid Tissue Kit (QIAGEN, Hilden, Germany) following the manufacturer's protocol. cDNA was synthesised from $2 \mu \mathrm{g}$ of total RNA using the TaqMan Reverse transcription reagent kit (Applied Biosystems, Foster City, CA, USA). A total of 20 ng of cDNA were subjected to quantitative RT-PCR amplification using a TaqMan Universal $2 \times$ PCR Master Mix (Applied Biosystems). Specific TaqManR Assay-On-Demand probes (Applied Biosystems) were used to study the level of expression for different genes: uncoupling protein 1 (UCP1; Rn 00562126_m1), PGC1 $\alpha$ (Rn 00580241_m1), Sirt1 (Rn 01428093_m1), nuclear respiratory factor 1 (Nrf 1; Rn 01455958_m1), COX5 $\alpha$ (Rn 00821806_m1)

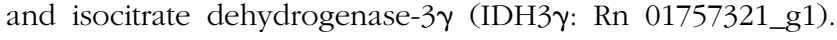
Peptidylprolyl isomerase A (cyclophilin A: Rn 00690933_m1) was used as an endogenous control. Real-time quantitative PCR were performed using the ABI Prism 7300 SDS Real-time PCR system (Applied Biosystems).

\section{Immunoblotting}

The relative quantification of UCP1 was measured in the mitochondrial fraction of BAT, previously used in respirometry. Western blot analysis was carried out according to Fuster et $a l^{(19)}$. Free ImageJ software (National Institutes of Health, Bethesda, MD, USA) was used to perform the relative quantification.

\section{Statistical analysis}

Results are reported as means with their standard errors. Group means were compared with an independent-sample Student's $t$ test $(P \leq 0.05)$ using SPSS for Windows version 17.0 software (SPSS, Inc., Chicago, IL, USA).

\section{Results \\ Grape seed proanthocyanidin extract treatment increases ketogenesis and slightly decreases the plasmatic concentration of TAG in obese rats}

Plasma parameters determined at the end point of the study do not indicate that GSPE supplementation significantly altered any parameter studied (Table 1). In both glucose and plasma lactate tests, there were no significant trends due to either the Cd or GSPE supplementation. Plasma TAG were shown to be notably increased $(P<0 \cdot 1)$ due to the administration of the Cd. GSPE administration tended to reduce circulating TAG levels, although this trend was not significant. In contrast, levels of ketone bodies showed a dose-dependent increase with GSPE supplementation, although the increase was not significant (Table 1).

Table 1. Plasma parameters of rats fed with a standard diet or cafeteria diet (Cd), either with or without proanthocyanidin administration*

(Mean values with their standard errors, $n 6$ )

\begin{tabular}{|c|c|c|c|c|c|c|c|c|}
\hline \multirow[b]{4}{*}{ Parameters } & \multicolumn{8}{|c|}{ Chronic dose } \\
\hline & & & & & & & & \\
\hline & \multicolumn{2}{|c|}{ Control } & \multicolumn{2}{|c|}{ Control Cd } & \multicolumn{2}{|c|}{$25 \mathrm{mg}$ GSPE $/ \mathrm{kg}$} & \multicolumn{2}{|c|}{$50 \mathrm{mg}$ GSPE $/ \mathrm{kg}$} \\
\hline & Mean & SEM & Mean & SEM & Mean & SEM & Mean & SEM \\
\hline Glucose (mg/l) & $1576 \cdot 1$ & $269 \cdot 6$ & $1943 \cdot 8$ & 163.6 & $2058 \cdot 1$ & $227 \cdot 6$ & $1642 \cdot 1$ & $162 \cdot 0$ \\
\hline 3-Hydroxybutyrate (mg/l) & $105 \cdot 5$ & $46 \cdot 0$ & $150 \cdot 6$ & $33 \cdot 1$ & $196 \cdot 7$ & $52 \cdot 6$ & 295.4 & $69 \cdot 6$ \\
\hline TAG $(\mathrm{mg} / \mathrm{l})$ & 958.9 & $45 \cdot 0$ & 2491.5 & 705.5 & 1801.6 & 358.5 & 1737.0 & $370 \cdot 8$ \\
\hline Lactate $(\mathrm{mg} / \mathrm{l})$ & $58 \cdot 3$ & $10 \cdot 1$ & $50 \cdot 6$ & $06 \cdot 8$ & 103.5 & $30 \cdot 1$ & 61.9 & $07 \cdot 7$ \\
\hline
\end{tabular}

GSPE, grape seed proanthocyanidin extract.

* Rats were fed with a standard chow diet (control group) or Cd (Cd plus standard chow diet) for 15 weeks. After 15 weeks, two groups of Cd rats were orally supplemented with 25 and $50 \mathrm{mg}$ GSPE/kg body weight and only the vehicle for the rest of the group during $21 \mathrm{~d}$. 


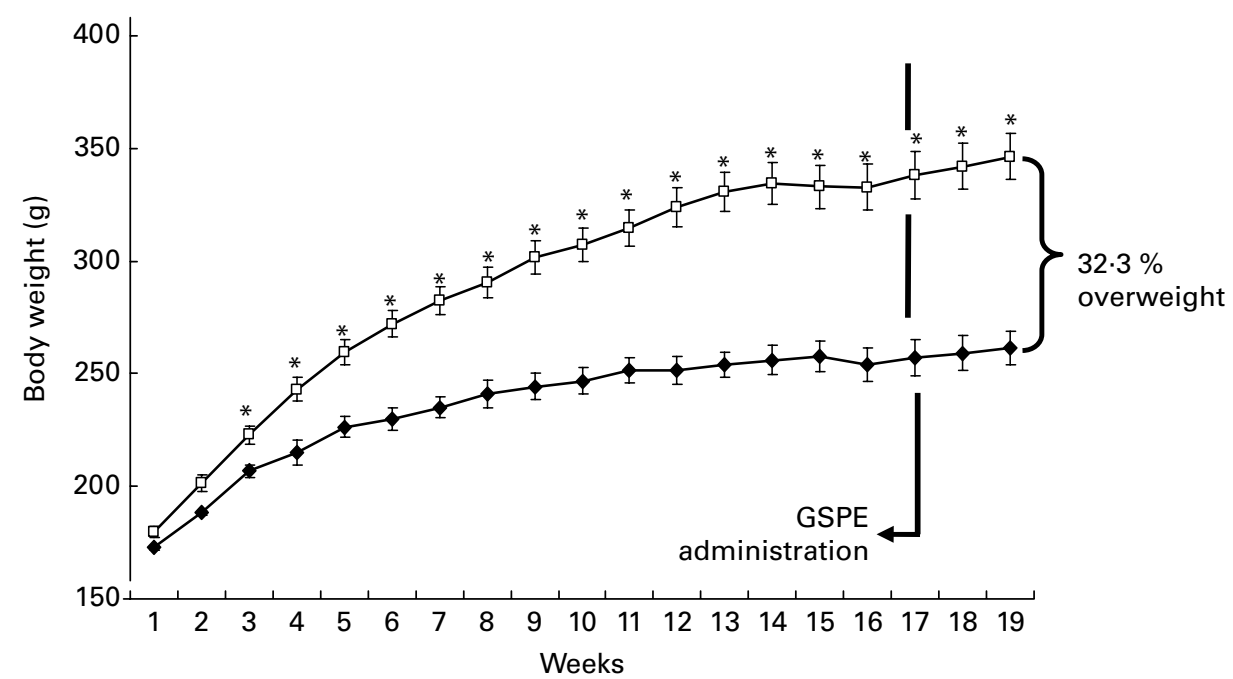

Fig. 1. Evolution of body weight. Rats were fed with a standard chow diet (control group, $-\downarrow-$ ) or cafeteria diet (cafeteria diet plus standard chow diet, $-\square-$ ) for 15 weeks. After 15 weeks, two groups of cafeteria diet rats were orally supplemented with 25 and 50 mg of grape seed proanthocyanidin extract (GSPE)/kg body weight and only the vehicle for the rest of the group during $21 \mathrm{~d}$. Values are means, with their standard errors represented by vertical bars $(n 6)$. ${ }^{*}$ Mean values were significantly different from those of the control group $(P<0.05)$.

\section{Grape seed proanthocyanidin extract administration} protects against weight gain in obesity induced by the cafeteria diet

Throughout the study, we monitored the evolution of body weight weekly. As expected, the results indicate that the $\mathrm{Cd}$ caused an increase in body weight of $32 \%$ after 19 weeks of administration (Fig. 1). Based on these data, we calculated the body-weight gain individually for each rat during the $21 \mathrm{~d}$ of treatment (Fig. 2). The results of this calculation showed an important finding that the dose $25 \mathrm{mg}$ GSPE/kg significantly decreased body-weight gain induced by the $\mathrm{Cd}$.

\section{Cafeteria diet significantly increased the size of brown} adipose tissue and the energy expenditure of obese rats but not the $R Q$

A week before starting rats on treatment with GSPE, we did indirect calorimetry on each rat to determine how the $\mathrm{Cd}$ affected the energy expenditure and RQ. There were no significant differences in RQ values between control and obese rats (data not shown), but we noted an increased energy expenditure in obese rats; in fact, we calculated the area under the curve and found a significant increase of $22.2 \%$ of energy expenditure for a $9 \mathrm{~h}$ measurement (Fig. 3), mainly due to an increase in BAT size produced by the $\mathrm{Cd}$ intake. We measured the weight of the entire BAT, and obese rats fed with the Cd increased $173 \%$ on average (Fig. 4).

\section{Grape seed proanthocyanidin extract chronic administration} produces an increase in the activity of cytochrome coxidase in the brown adipose tissue of obese rats

To assess whether dietary supplementation of GSPE affecting enzymes involved in the citric acid cycle and oxidative phosphorylation in BAT, we measured the enzymatic activities of citrate synthase, COX and ATPase in freshly isolated mitochondria (Table 2).
The results show that obesity induced by the Cd caused an important decrease in COX activity. Although it was not significantly, the administration of GSPE interestingly tended to increase the COX activity in a dose-dependent manner. The results of citrate synthase activity of BAT showed that the activity tended to decrease with increased obesity and with the administration of GSPE, although no significant differences were found. The ATPase activity of BAT does not seem to be affected either by obesity or treatment with GSPE (Table 2).

\section{Chronic supplementation of grape seed proanthocyanidin extract corrects the gene expression of key genes in the brown adipose tissue mitochondria caused by obesity}

To determine whether chronic administration of GSPE modifies the gene expression of key genes in energy metabolism

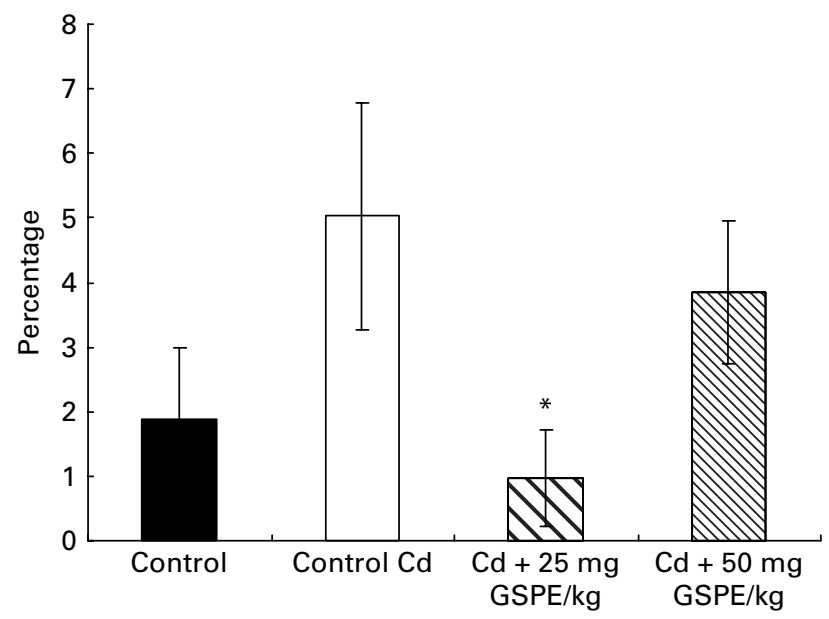

Fig. 2. Percentage of body-weight gain during grape seed proanthocyanidin extract (GSPE) administration. Values are the percentage of variation of body weight during the $21 \mathrm{~d}$ of GSPE administration. Values are means, with their standard errors represented by vertical bars $(n 6) .{ }^{*}$ Mean values were significantly different from those of the control cafeteria diet $(\mathrm{Cd})$ group $(P<0.05)$. 


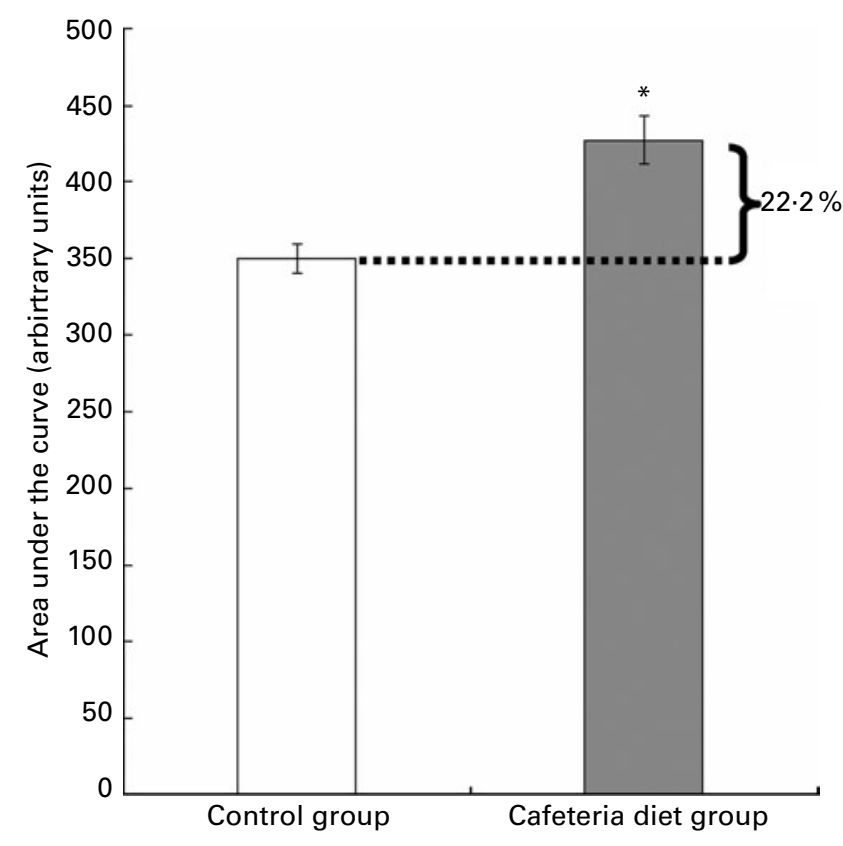

Fig. 3. Energy expenditure during $9 \mathrm{~h}$. Arbitrary units of area under the curve for $9 \mathrm{~h}$ of the average values of energy expenditure of each group. This measurement was made before the period of grape seed proanthocyanidin extract administration. Values are means, with their standard errors represented by vertical bars. * Mean values were significantly different from those of the control group $(P<0.05)$.

and mitochondrial function, such as PGC1 $\alpha$, Sirt1 or UCP1 among other genes, we perform an RT-PCR of the BAT.

The present gene expression results indicate that obesity induced by diet significantly suppressed the expression of important genes involved in mitochondrial function, such as Sirt1, and Nrf 1, as well as those involved in both the citric acid cycle, such as IDH3 $\gamma$, and the electron transport chain, for which we use $\operatorname{COX} 5 \alpha$ expression as a marker. Other important genes such as UCP1 and PGC1 $\alpha$ decreased with obesity, but not significantly. Interestingly, this repression of gene expression caused by obesity was corrected to the level of controls by chronic administration of GSPE in almost all genes studied. It is noteworthy that $\mathrm{Nrf} 1$ is one of the few genes studied that did not follow the profile of other genes. In other words, Nrf 1 does not seem to be affected by chronic administration of GSPE. While PGC1 $\alpha$ did not show any significant differences between the groups, there is a clear increase in gene expression upon administration of GSPE.

\section{Chronic administration of grape seed proanthocyanidin extract enhances mitochondrial respiration with both pyruvate and carnitine-palmitoyl-CoA as substrates}

The levels of each state of mitochondrial respiration, with both pyruvate and carnitine-palmitoyl-CoA as substrates, dramatically decrease the levels of respiration and thus mitochondrial function in cafeteria-diet-induced obese rats (Fig. 5(A) and (B), respectively). ADP-stimulated respiration (state 3), oligomycin-insensitive rate of respiration (state 4) and $P$-trifluoromethoxyphenylhydrazone-stimulated respiration (uncoupled state) were significantly different using pyruvate as a substrate between the control and control Cd groups, mainly due to obesity. However, the Cd groups that were supplemented with GSPE showed higher levels of mitochondrial respiration, and therefore better mitochondrial function, in all mitochondrial states than the controls (Fig. 5(A)). In the case where we use carnitine-palmitoyl-CoA as a substrate, the present results also showed a diminished ability to degrade a lipid substrate in obese rats. This mitochondrial dysfunction caused, in part, by obesity induced by diet was more pronounced in state 2 with carnitine and in the uncoupled state. Similarly, we also observed a significant increase in respiration levels and consequently, an improvement in the oxidation of fatty acids in the groups that were supplemented with GSPE (Fig. 5(B)).

\section{Grape seed proanthocyanidin extract administration slightly increased the amount of uncoupling protein 1}

The results obtained by Western blot show no significant differences in the amount of UCP1 protein between the groups. The diet-induced obesity slightly increased the amount of UCP1, and the groups that were supplemented with GSPE showed a slight tendency to increase the amount of UCP1, but neither case was significant.

\section{Discussion}

There are many studies about the beneficial effects that proanthocyanidin consumption produces in the physiology and biochemistry of organisms that consume it ${ }^{(13,15,16,25)}$, but few studies have been done to determine the effect of proanthocyanidins on energy metabolism ${ }^{(26)}$. Here, we have tried to evaluate the role of proanthocyanidins and their interaction with the control mechanisms of body weight by studying the effect of proanthocyanidins on the function of BAT mitochondria in a model of obesity induced by diet.

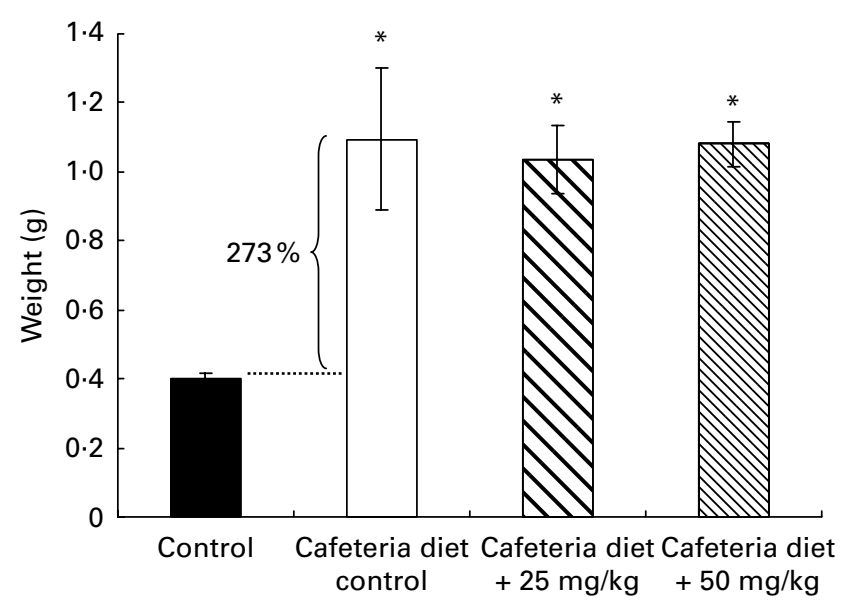

Fig. 4. Brown adipose tissue weight. These values represent the brown adipose tissue weight. Values are means, with their standard errors represented by vertical bars. ${ }^{*}$ Mean values were significantly different from those of the control group $(P<0.05)$. 
Table 2. Total activity (nkat/g tissue) of the mitochondrial fraction from the brown adipose tissue of rats fed with a standard diet or cafeteria diet $(\mathrm{Cd})$, either with or without proanthocyanidin administration* (Mean values with their standard errors, $n 6$ )

\begin{tabular}{|c|c|c|c|c|c|c|c|c|}
\hline \multirow[b]{4}{*}{ Enzymatic activities } & \multicolumn{8}{|c|}{ Chronic dose } \\
\hline & \multirow{2}{*}{\multicolumn{2}{|c|}{ Control }} & \multicolumn{6}{|c|}{$\mathrm{Cd}$} \\
\hline & & & \multicolumn{2}{|c|}{ Control Cd } & \multicolumn{2}{|c|}{25 mg GSPE/kg } & \multicolumn{2}{|c|}{$\begin{array}{c}50 \mathrm{mg} \\
\text { GSPE } / \mathrm{kg}\end{array}$} \\
\hline & Mean & SEM & Mean & SEM & Mean & SEM & Mean & SEM \\
\hline Citrate synthase & 44.21 & 11.58 & 39.95 & 7.75 & 31.42 & $3 \cdot 17$ & $26 \cdot 80$ & 3.65 \\
\hline $\operatorname{cox}$ & $70 \cdot 71$ & 21.83 & $42 \cdot 34$ & 7.57 & $48 \cdot 70$ & 14.25 & $56 \cdot 94$ & 2.33 \\
\hline ATPase & $5 \cdot 38$ & 0.50 & 5.65 & 0.96 & 4.89 & 0.83 & $5 \cdot 27$ & 0.65 \\
\hline
\end{tabular}

GSPE, grape seed proanthocyanidin extract; COX, cytochrome $c$ oxidase. * Experimental details are in Table 1.

There was a clear increase of $32 \%$ body weight in dietinduced obesity rats, and it was linked to hypertrophy of interscapular BAT reaching up to a $170 \%$ increase in magnitude (similar to the increases found by other authors ${ }^{(27-30)}$ ). Moreover, the relationship between protein content and BAT weight was constant, due to an increase in the number of brown adipocytes ${ }^{(28,31,32)}$.
Diet-induced obesity causes an increase in energy expenditure $^{(33)}$ that, according to our indirect calorimetry results, is estimated to increase about $22 \%$ for $9 \mathrm{~h}$. This increase in energy expenditure in basal conditions can be attributed to an increased production of ATP, which correlates with an increase in the amount of BAT and UCP1 protein, in order to generate heat and counteract the development of obesity ${ }^{(34-36)}$.

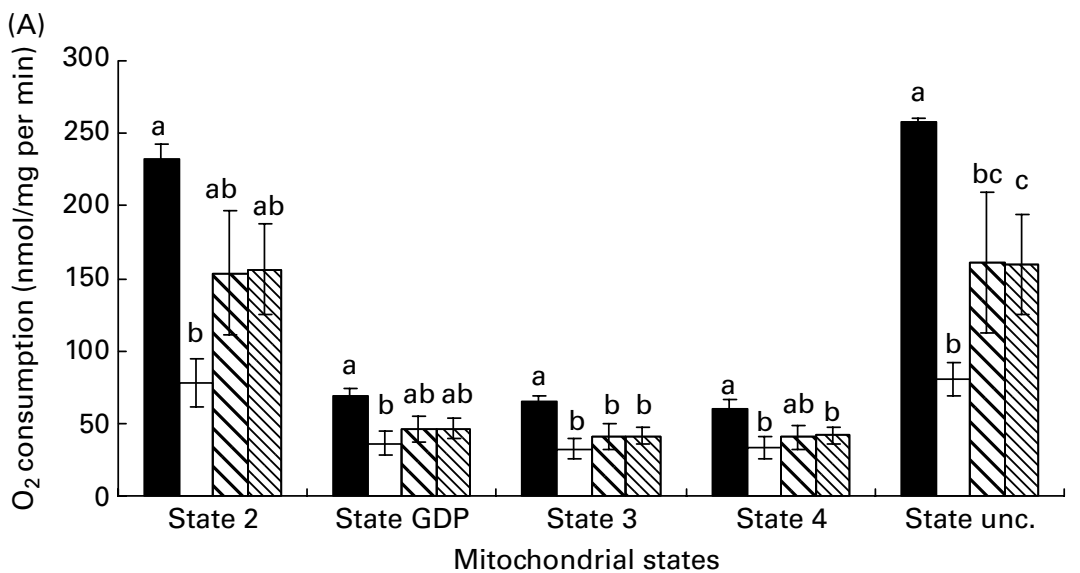

(B)

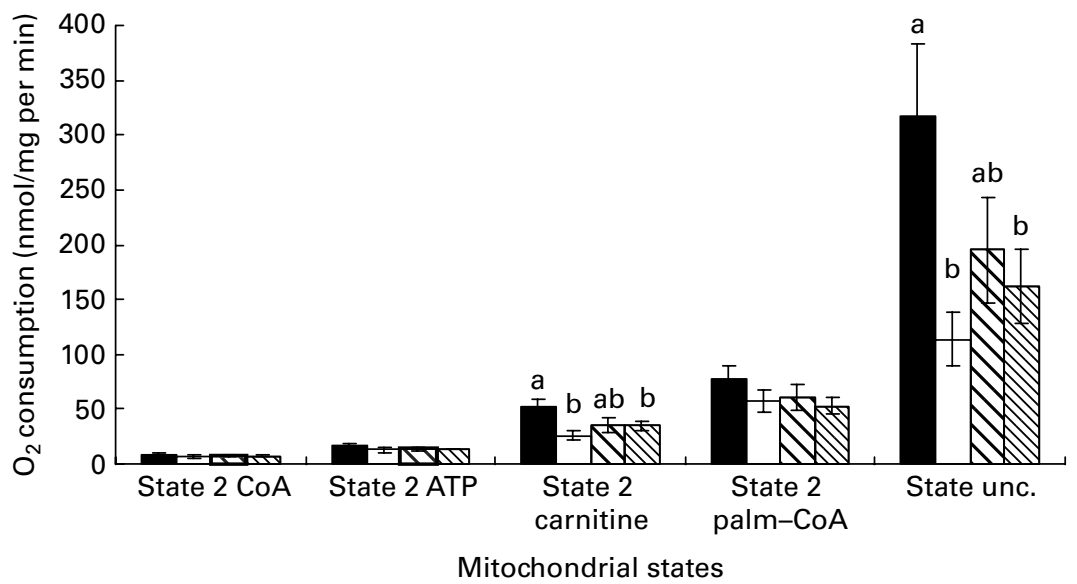

Fig. 5. Brown adipose tissue (BAT) high-resolution respirometry. $\mathrm{O}_{2}$ consumption due to oxidative phosphorylation in different mitochondrial states from $B A T$ mitochondria incubated with (A) pyruvate and (B) carnitine-palmitoyl-CoA substrates. Values are means, with their standard errors represented by vertical bars $(n 6) .{ }^{a, b, c}$ Mean values with unlike letters were significantly different between the groups $(P<0.05$; Student's $t$ test). $\mathbf{m}$, Control; $\square$, control cafeteria diet $(C d)$; $\varangle, \mathrm{Cd}+25 \mathrm{mg}$ GSPE $/ \mathrm{kg} ; \otimes, \mathrm{Cd}+50 \mathrm{mg}$ GSPE$/ \mathrm{kg}$. GSPE, grape seed proanthocyanidin extract; palm-CoA, palmitoyl-CoA; state unc., uncoupled state. 
Increases in BMI and visceral fat mass are associated with obesity, which is associated with a lower activity of BAT ${ }^{(37)}$ and therefore a lower thermogenic capacity. Conversely, individuals who have lower activity or function of BAT and therefore lower thermogenic capacity are probably prone to develop obesity ${ }^{(38)}$.

The decrease in thermogenic capacity and the increase in energy expenditure that occurs in obesity could be controlled by increasing the size of BAT and changing the concentration and activity of UCP1. The present results indicate that obesity induced by diet does not change the transcriptional level of UCP1 but does produce an increase in the amount of UCP1 in BAT. This increase was found to be insignificant, but is interesting considering the significant increase in the size of BAT in obese rats. Some authors have shown similar results $^{(28,29)}$, while others have detected an increase in the transcription of the UCP1 gene ${ }^{(1,39,40)}$.

Age is one of the causes of a decrease in diet-induced thermogenesis, but despite this, obesity is associated with an increase in the concentration of UCP1 in $\mathrm{BAT}^{(41)}$. Diet can also modify the activity of UCP1 because it has been shown that diets rich in $n-3$ PUFA cause an increase in the thermogenic capacity of BAT without altering the content of $\mathrm{UCP} 1^{(29)}$.

Although there is much more UCP1 protein in a state of obesity induced by diet because of a hypertrophy of BAT, in the present analysis, obesity produced a strong decrease in UCP1 activity in BAT. The results from the high-resolution respirometry using pyruvate as a substrate clearly showed how diet-induced obesity leads to a $66 \%$ decrease in UCP1 activity (state 2). Initially, the mitochondria isolated from BAT were uncoupled, and the increase in respiration that we observed in state 2 was due to the addition of pyruvate. Because this increase in respiration in state 2 can be inhibited by the addition of GDP, the respiration is mediated by the activity of $\mathrm{UCP} 1^{(20)}$. Moreover, not only the activity of UCP1 decreased with increasing obesity, but also the overall mitochondrial function and activity because all other mitochondrial states tested were also diminished.

It is commonly thought that thermogenesis of BAT is due exclusively to the combustion of lipids, but the values of the respiratory coefficients of animals in cold environments indicate the combined utilisation of both carbohydrates and lipids $^{(42)}$. Even the ability to oxidise substrates from a carbohydrate, such as pyruvate, is better than the ability to oxidise fatty acids, such as carnitine-palmitoyl-CoA ${ }^{(42)}$, and, in this sense, we have also studied the mitochondrial function with a lipid substrate. To this end, we used a protocol previously published by Cannon ${ }^{(22)}$ to determine the ability of fatty acid oxidation in BAT mitochondria. In this case, we added ATP, coenzyme A and carnitine to promote the recoupling of the mitochondria. We continued with the addition of carnitine, resulting in increased mitochondrial respiration due to combustion of an endogenous substrate of mitochondria. After the addition of palmitoyl-CoA, we observed an increase in respiration due to the combustion of palmitoyl-CoA. $p$-Trifluoromethoxyphenylhydrazone, a chemical uncoupler, was added to allow observation of the maximum mitochondrial capacity using this substrate. The results also showed that obesity induced by diet reduced the capacity of fatty acid oxidation, which is most clearly expressed during the uncoupled state.

Moreover, these results suggest that obesity induced by diet causes mitochondrial dysfunction in BAT that is correlated with a decrease in the expression of key genes of energy metabolism. Sirt1 deacetylates and activates the transcription factor PGC1 $\alpha$, which controls mitochondrial function and adaptive thermogenesis in $\mathrm{BAT}^{(9)}$. The present results showed a decreased transcription of the genes encoding Sirt1 and PGC1 $\alpha$ proteins during a state of obesity. This decrease can explain all of the phenomena that we observed in the respiration of mitochondria from BAT in a situation of obesity.

The BAT of obese rats showed decreased gene expression of both IDH $3 \gamma$, a regulatory enzyme of the citric acid cycle, and $\operatorname{COX} 5 \alpha$, subunit 5 of complex IV in the mitochondrial electron transport chain. This last finding agrees with previously published obesity studies ${ }^{(31)}$. The decrease in the expression of $\operatorname{COX} 5 \alpha$ that we observed correlated with a decrease in the enzyme activity of COX; however, the change was not quite significant.

All of these data clearly demonstrate that obesity induced by the Cd causes a significant decrease in mitochondrial activity and functionality at all levels in BAT.

Proanthocyanidins have been shown to have an ability to produce a beneficial effect on the health of those organisms that ingest them ${ }^{(13,15,16,25)}$. In the present study, we present evidence to support the hypothesis that the proanthocyanidins can affect the mitochondrial function of BAT and, therefore, its thermogenic capacity, after a decrease in both function and capacity induced by obesity.

Previous evidence from the literature suggests that the prolonged consumption of polyphenols from natural extracts improves the functionality of BAT. Specifically, Lagouge et $a l^{(9)}$ suggested that the administration of $0.4 \%$ resveratrol in chow causes a significant increase in the mitochondrial activity of BAT in mice. In agreement with this finding, Dulloo et $a .^{(43)}$ indicated that the catechins of green tea extract contain thermogenic properties and promote fat oxidation in human subjects. In addition, dietary supplementation of $0.5 \%$ of catechins from green tea in chow led to an increase in the amount of UCP1 in Sprague-Dawley rats, although this did not occur in those rats that had been fed with a high-fat diet ${ }^{(1)}$. In vitro studies have shown that $(-)$ epigallocatechin gallate inhibits catechol-O-methyltransferase, an enzyme which degrades noradrenaline ${ }^{(44)}$. This extension of nonadrenaline signalling stimulates $\beta$-adrenergic receptors that produce an increase in the thermogenic capacity of $\mathrm{BAT}^{(4)}$.

In this context, we wished to study the possible effects of a chronic GSPE dietary supplementation on mitochondrial function of BAT because until now very little was known about the ability of proanthocyanidins to stimulate mitochondrial function and BAT thermogenic capacity in vivo.

All of the present results indicate that chronic administration of GSPE improves mitochondrial function and, therefore, the thermogenic capacity of BAT. Specifically, we have observed that supplementation of GSPE tended to correct the decrease 
in Sirt1 and PGC1 $\alpha$ gene expression caused by obesity, suggesting that GSPE administration leads to improved mitochondrial function and thermogenic capacity in the BAT of obese rats. This improvement caused by chronic GSPE supplementation was also reflected in the higher levels of the gene expression of IDH3 $\gamma$ and $\mathrm{COX} 5 \alpha$, suggesting an improvement of both the functionality of the citric acid cycle and the electron transport chain in the BAT of obese rats.

These gene expression results were correlated with increased COX activity, although this was not significant. Moreover, these results are consistent with Lagouge et al. ${ }^{(9)}$ who indicated that administration of $0.4 \%$ of resveratrol in chow improves mitochondrial function in the BAT of mice that were fed a high-fat diet and significantly increases the expression and deacetylation of PGC1 $\alpha$ by Sirt1 activity. They also observed an increased expression of UCP1 due to the administration of resveratrol. Interestingly, we also observed a significant increase in the gene expression of UCP1 in the BAT of obese rats supplemented with $25 \mathrm{mg}$ GSPE/kg body weight. Our immunoblotting results indicate a tendency to increase the amount of UCP1 protein, although this increase was not significant. These results suggest a greater thermogenic capacity of BAT in rats that were administered GSPE than that of obese rats that were not supplemented with GSPE.

Beyond these findings, the results of high-resolution respirometry performed in isolated mitochondria from BAT using pyruvate as a substrate revealed that GSPE administration enhances the activity of UCP1 (state 2). Not only was UCP1 activity increased with GSPE administration, but also the activity of oxidative phosphorylation in general because GSPE improved the respiration levels of all mitochondrial states using pyruvate as a substrate. The improvement of mitochondrial function is evident in the combustion of pyruvate and is also evident in the oxidation of carnitine-palmitoylCoA. These results suggest that the increased activity of oxidative phosphorylation corrects the dysfunction caused by obesity induced by diet.

In fact, the mitochondrial effects induced by GSPE supplementation that we observed at the transcriptional and activity level are correlated with decreased body-weight gain. The results of the difference in body weight during the $21 \mathrm{~d}$ of GSPE supplementation revealed that rats that have been supplemented with GSPE, especially at a dose of $25 \mathrm{mg} / \mathrm{kg}$, significantly reduced their body-weight gain despite being fed with the $\mathrm{Cd}$, until it reached values similar to those of the control rats. Therefore, obesity induced by diet reduces the thermogenic capacity of BAT, but obese rats that were administrated with GSPE were protected against weight gain, partly due to the improved mitochondrial function of BAT and, consequently, an increased thermogenic capacity.

Taken together, our data indicate that chronic administration of GSPE enhances thermogenic capacity and improves mitochondrial function in the BAT of cafeteria-diet-induced obese rats. In addition, these effects appear to be associated with protection against obesity induced by the $\mathrm{Cd}$. The present study aims to shed light on the use of GSPE and other natural compounds that stimulate energy expenditure in BAT as a therapy against obesity.

\section{Acknowledgements}

The present study was supported by the Spanish Government, Secretaria de Estado de Investigación del Ministerio de Ciencia, financing the project AGL2008-00387/ALI. We would like to thank N. Llópiz for her technical assistance, and the MITOFOOD COST network of which L. A. is a member. C. B., J. S. and L. A. had full access to all of the data in the study and were responsible for the integrity of the data and the accuracy of the data analysis. C. B., J. S. and L. A. were involved in the study concept and design. D. P., H. Q., S. D. and A. F.-I. were involved in the acquisition of the data. D. P., H. Q., S. D., A. F.-I., A. A.-A., C. B., J. S. and L. A. performed the analyses and interpretation of the data. D. P. and L. A. drafted the manuscript. D. P., H. Q., S. D., A. F.-I., A. A.-A., C. B., J. S. and L. A. contributed to the critical revision of the manuscript for important intellectual content. D. P., A. A.-A. and L. A. provided administrative, technical and logistic support. The authors declare that there is no conflict of interest.

\section{References}

1. Nomura S, Ichinose T, Jinde M, et al. (2008) Tea catechins enhance the mRNA expression of uncoupling protein 1 in rat brown adipose tissue. J Nutr Biochem 19, 840-847.

2. Fruhbeck G, Becerril S, Sainz N, et al. (2009) BAT: a new target for human obesity? Trends Pharmacol Sci 30, 387-396.

3. Hansen JB \& Kristiansen K (2006) Regulatory circuits controlling white versus brown adipocyte differentiation. Biochem J 398, $153-168$

4. Cannon B \& Nedergaard J (2004) Brown adipose tissue: function and physiological significance. Physiol Rev $\mathbf{8 4}$ $277-359$

5. Kozak LP \& Anunciado-Koza R (2008) UCP1: its involvement and utility in obesity. Int $J$ Obes (Lond) 32, Suppl. 7, S32-S38.

6. van Marken Lichtenbelt WD, Vanhommerig JW, Smulders NM, et al. (2009) Cold-activated brown adipose tissue in healthy men. $N$ Engl J Med 360, 1500-1508.

7. Virtanen KA, Lidell ME, Orava J, et al. (2009) Functional brown adipose tissue in healthy adults. $N$ Engl J Med $\mathbf{3 6 0}$, 1518-1525.

8. Cypess AM, Lehman S, Williams G, et al. (2009) Identification and importance of brown adipose tissue in adult humans. $N$ Engl J Med 360, 1509-1517.

9. Lagouge M, Argmann C, Gerhart-Hines Z, et al. (2006) Resveratrol improves mitochondrial function and protects against metabolic disease by activating SIRT1 and PGC1alpha. Cell 127, 1109-1122.

10. Lopez-Lluch G, Hunt N, Jones B, et al. (2006) Calorie restriction induces mitochondrial biogenesis and bioenergetic efficiency. Proc Natl Acad Sci USA 103, 1768-1773.

11. Davis JM, Murphy EA, Carmichael MD, et al. (2009) Quercetin increases brain and muscle mitochondrial biogenesis and exercise tolerance. Am J Physiol Regul Integr Comp Physiol 296, R1071-R1077.

12. Rasmussen SE, Frederiksen H, Struntze Krogholm K, et al. (2005) Dietary proanthocyanidins: occurrence, dietary 
intake, bioavailability, and protection against cardiovascular disease. Mol Nutr Food Res 49, 159-174.

13. Blade C, Arola L \& Salvado MJ (2010) Hypolipidemic effects of proanthocyanidins and their underlying biochemical and molecular mechanisms. Mol Nutr Food Res 54, 37-59.

14. Puiggros F, Llopiz N, Ardevol A, et al. (2005) Grape seed procyanidins prevent oxidative injury by modulating the expression of antioxidant enzyme systems. J Agric Food Chem 53, 6080-6086.

15. Terra X, Montagut G, Bustos M, et al. (2009) Grape-seed procyanidins prevent low-grade inflammation by modulating cytokine expression in rats fed a high-fat diet. $J$ Nutr Biochem 20, 210-218.

16. Quesada H, del Bas JM, Pajuelo D, et al. (2009) Grape seed proanthocyanidins correct dyslipidemia associated with a high-fat diet in rats and repress genes controlling lipogenesis and VLDL assembling in liver. Int $J$ Obes (Lond) $\mathbf{3 3}$, 1007-1012.

17. Rasbach KA \& Schnellmann RG (2008) Isoflavones promote mitochondrial biogenesis. J Pharmacol Exp Ther 325, 536-543.

18. Caimari A, Oliver P, Rodenburg W, et al. (2010) Feeding conditions control the expression of genes involved in sterol metabolism in peripheral blood mononuclear cells of normoweight and diet-induced (cafeteria) obese rats. $J$ Nutr Biochem 21, 1127-1133.

19. Fuster A, Oliver P, Sanchez J, et al. (2009) UCP1 and oxidative capacity of adipose tissue in adult ferrets (Mustela putorius furo). Comp Biochem Physiol A Mol Integr Physiol 153, 106-112.

20. Cannon B \& Nedergaard J (2008) Studies of thermogenesis and mitochondrial function in adipose tissues. Methods $\mathrm{Mol}$ Biol 456, 109-121.

21. Udenfriend S, Stein S, Bohlen P, et al. (1972) Fluorescamine: a reagent for assay of amino acids, peptides, proteins, and primary amines in the picomole range. Science $\mathbf{1 7 8}$, $871-872$

22. Cannon B (1971) Control of fatty-acid oxidation in brownadipose-tissue mitochondria. Eur J Biochem 23, 125-135.

23. Srere PA (1969) Citrate Synthase. In Methods in Enzymology, vol. 13, pp. 3-11 [JM Lowenstein, editor]. New York, NY: Academic Press.

24. Penefsky HS \& Bruist MF (1984) Adenosine triphosphatases. In Methods of Enzymatic Analysis, vol. 4, pp. 324-328 [HU Bergmeyer, J Bergmeyer and M Grabl, editors]. Weinheim, Germany: Verlag Chemie GMbH.

25. Del Bas JM, Fernandez-Larrea J, Blay M, et al. (2005) Grape seed procyanidins improve atherosclerotic risk index and induce liver CYP7A1 and SHP expression in healthy rats. FASEB J 19, 479-481.

26. Zheng J \& Ramirez VD (2000) Inhibition of mitochondrial proton F0F1-ATPase/ATP synthase by polyphenolic phytochemicals. Br J Pharmacol 130, 1115-1123.

27. Sene-Fiorese M, Duarte FO, Scarmagnani FR, et al. (2008) Efficiency of intermittent exercise on adiposity and fatty liver in rats fed with high-fat diet. Obesity (Silver Spring) 16, 2217-2222.

28. Rodriguez E, Monjo M, Rodriguez-Cuenca S, et al. (2001) Sexual dimorphism in the adrenergic control of rat brown adipose tissue response to overfeeding. Pflugers Arch $\mathbf{4 4 2}$, 396-403.

29. Harrold JA, Widdowson PS, Clapham JC, et al. (2000) Individual severity of dietary obesity in unselected Wistar rats: relationship with hyperphagia. Am J Physiol Endocrinol Metab 279, E340-E347.

30. Roca P, Rodriguez AM, Oliver P, et al. (1999) Brown adipose tissue response to cafeteria diet-feeding involves induction of the UCP2 gene and is impaired in female rats as compared to males. Pflugers Arch 438, 628-634.

31. Rodriguez AM, Quevedo-Coli S, Roca P, et al. (2001) Sexdependent dietary obesity, induction of UCPs, and leptin expression in rat adipose tissues. Obes Res 9, 579-588.

32. Rodriguez AM, Roca P \& Palou A (2002) Synergic effect of overweight and cold on uncoupling proteins expression, a role of alpha(2)/beta(3) adrenergic receptor balance? Pflugers Arch 444, 484-490.

33. Jackman MR, MacLean PS \& Bessesen DH (2010) Energy expenditure in obesity-prone and obesity-resistant rats before and after the introduction of a high-fat diet. $A m J$ Physiol Regul Integr Comp Physiol 299, R1097-R1105.

34. Fromme $\mathrm{T} \&$ Klingenspor M (2011) Uncoupling protein 1 expression and high fat diets. Am J Physiol Regul Integr Comp Physiol 300, R1-R8.

35. Rodriguez AM \& Palou A (2004) Uncoupling proteins: gender dependence and their relation to body weight control. Int J Obes Relat Metab Disord 28, 500-502.

36. Rodriguez E, Ribot J, Rodriguez AM, et al. (2004) PPARgamma2 expression in response to cafeteria diet: genderand depot-specific effects. Obes Res 12, 1455-1463.

37. Saito M, Okamatsu-Ogura Y, Matsushita M, et al. (2009) High incidence of metabolically active brown adipose tissue in healthy adult humans: effects of cold exposure and adiposity. Diabetes 58, 1526-1531.

38. Mattson MP (2010) Perspective: does brown fat protect against diseases of aging? Ageing Res Rev 9, 69-76.

39. Ricquier D \& Bouillaud F (2000) The uncoupling protein homologues: UCP1, UCP2, UCP3, StUCP and AtUCP. Biochem J 345, Pt 2, 161-179.

40. Perez-Echarri N, Noel-Suberville C, Redonnet A, et al. (2007) Role of adipogenic and thermogenic genes in susceptibility or resistance to develop diet-induced obesity in rats.J Physiol Biochem 63, 317-327.

41. Rothwell NJ \& Stock MJ (1983) Effects of age on diet-induced thermogenesis and brown adipose tissue metabolism in the rat. Int J Obes 7, 583-589.

42. Cannon B \& Nedergaard J (2010) Metabolic consequences of the presence or absence of the thermogenic capacity of brown adipose tissue in mice (and probably in humans). Int J Obes (Lond) 34, Suppl. 1, S7-S16.

43. Dulloo AG, Duret C, Rohrer D, et al. (1999) Efficacy of a green tea extract rich in catechin polyphenols and caffeine in increasing 24-h energy expenditure and fat oxidation in humans. Am J Clin Nutr 70, 1040-1045.

44. Lu H, Meng X \& Yang CS (2003) Enzymology of methylation of tea catechins and inhibition of catechol-O-methyltransferase by (-)-epigallocatechin gallate. Drug Metab Dispos 31 , $572-579$. 